\title{
Gastric Volvulus: A Rare Diagnosis of Abdominal Pain
}

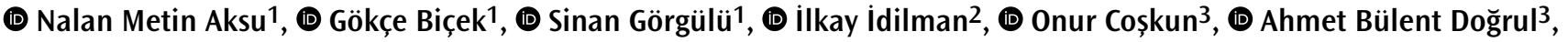 \\ (1) Meltem Akkaș1
}

1Department of Emergency Medicine, Hacettepe University Faculty of Medicine, Ankara, Turkey

2Department of Radiology, Hacettepe University Faculty of Medicine, Ankara, Turkey

${ }^{3}$ Department of General Surgery, Hacettepe University Faculty of Medicine, Ankara, Turkey

\begin{abstract}
Gastric volvulus (GV) is a rare but life-threatening condition in case of delay in diagnosis and treatment. Herein, we presented a case of GV due to Morgagni hernia presenting to the emergency department with complaints of abdominal pain and distention. A 59-year-old male patient was admitted to the emergency department with abdominal pain and distension, nausea and vomiting. His past medical history included coronary artery disease, hypertension, multiple sclerosis and left diaphragmatic hernia. On initial examination, pulse rate was 119/min and other vital signs were normal. Abdominal examination revealed epigastric tenderness. Posteroanterior chest X-ray showed an air bubble in the left hemithorax. Thoracoabdominal CT was performed for differential diagnosis. Department of Emergency Radiology reported a mesenteroaxial gastric volvulus at the superior gastro-esophageal junction with a Morgagni hernia including left hemidiaphragmatic defect with herniation of the gastric corpus, first part of the duodenum and transverse colon. Following placement of the nasogastric tube, $1300 \mathrm{~mL}$ gastric secretion was drained and abdominal distention was relieved. Patient was referred to the operating room. Morgagni hernia and the diaphragm defect were repaired. During follow-up in general surgery ward, a sudden cardiac arrest developed on the $5^{\text {th }}$ day and he died. If a patient presents to the emergency departmen with abdominal pain and distention, GV should be considered in the differential diagnosis, although rare.
\end{abstract}

Keywords: Abdominal pain, abdominal distention, gastric volvulus, emergency department

\section{Introduction}

Gastric volvulus (GV) is a rare but life-threatening condition in case of delayed diagnosis and treatment. It is defined as abnormal rotation of the stomach more than 180 degrees. Risk factors for GV in adults include: age greater than 50 years, diaphragmatic abnormalities (diaphragmatic injury and eventration, left lung resection or pleural adhesions), phrenic nerve paralysis, other anatomical gastrointestinal pyloric stenosis, gastroduodenal tumors or splenic abnormalities and kyphoscoliosis $(1,2)$. Herein, we present a case of GV due to Morgagni hernia presenting to the emergency department with complaints of abdominal pain and distention.

\section{Case Report}

A 59-year-old male patient was admitted to the emergency department with symptoms of abdominal pain and distension, and nausea and vomiting. His past medical history included coronary artery disease, hypertension, multiple sclerosis and left diaphragmatic hernia. On initial examination, pulse rate was $119 / \mathrm{min}$, and other vital signs were normal. Abdominal examination revealed epigastric tenderness. His white blood count was $17800 / \mathrm{mm}^{3}$ and potassium was $3 \mathrm{mEq} / \mathrm{lt}$. Other blood count parameters and biochemistry parameters were within normal ranges. Posteroanterior chest $\mathrm{X}$-ray showed an air bubble in the left hemithorax (Figure 1). Thoracoabdominal CT was performed for differential diagnosis. Department of emergency 
radiology reported a mesenteroaxial GV located at the superior gastro-esophageal junction with a Morgagni hernia including left hemidiaphragmatic defect with herniation of the gastric corpus, first part of the duodenum and transverse colon (Figure 2). The patient was consulted with general surgery clinic. Following placement of the nasogastric tube, $1300 \mathrm{~mL}$ gastric secretion was drained and abdominal distention was relieved. The patient was referred to the operating room. Morgagni hernia and the diaphragmatic defect were repaired. During follow-up in his

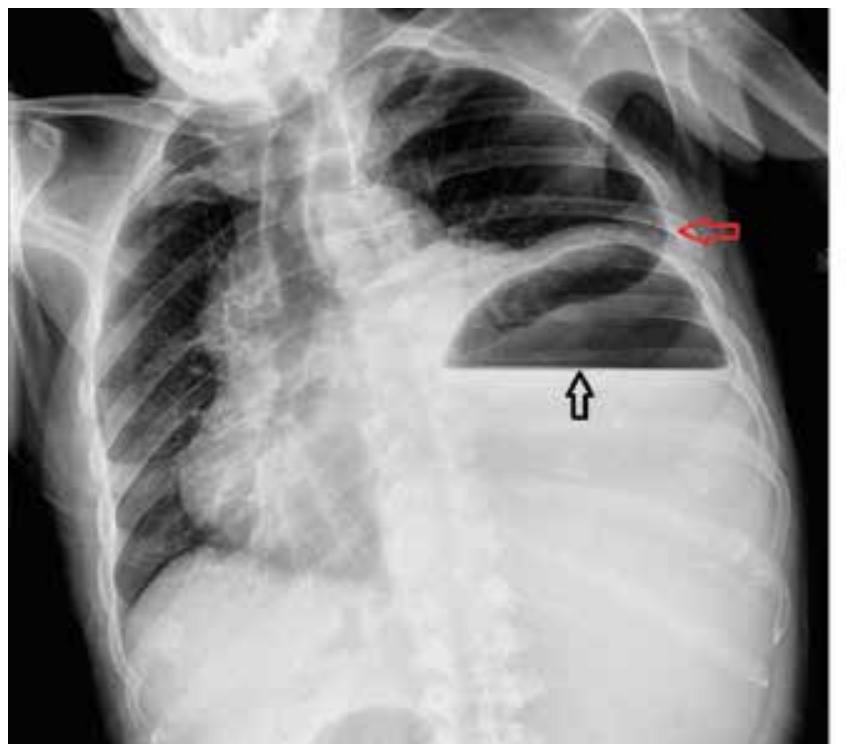

Figure 1. An air bubble in the left hemithorax on posteroanterior chest X-ray

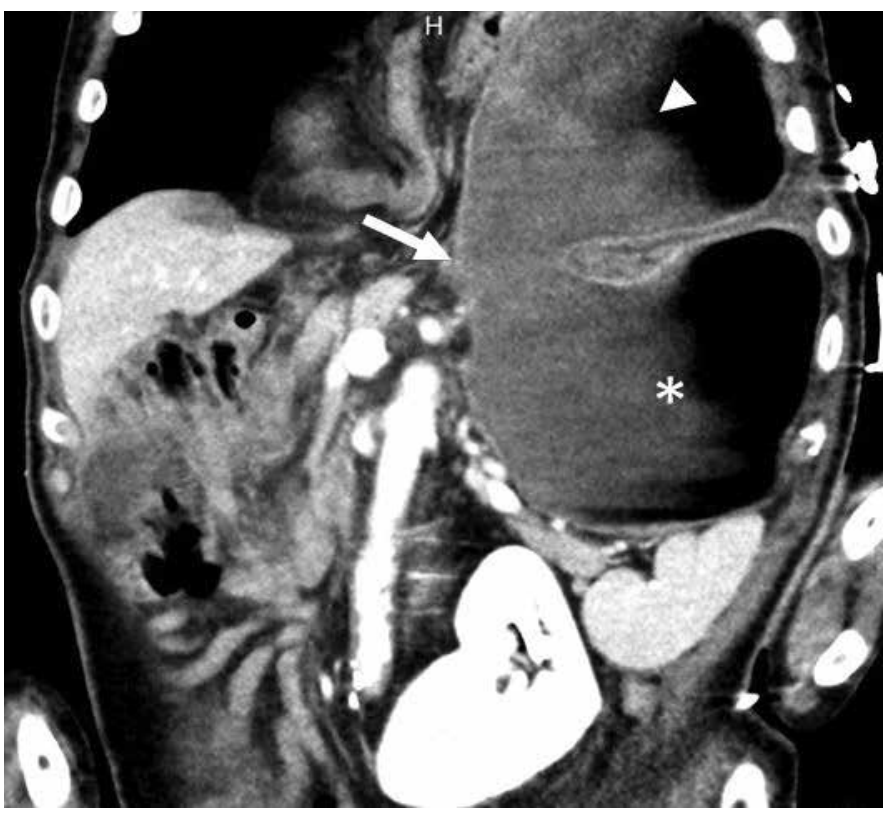

Figure 2. Coronal computed tomography multiplanar reformatted image demonstrates the displacement of the antrum above gastroesophageal junction level (white arrow) with antrum and pylorus (arrow head located superior to the fundus and proximal body $\left(^{*}\right.$ ) observation in the general surgery ward, a sudden cardiac arrest developed on the $5^{\text {th }}$ day and he died. The surgeons anticipated that cardiac arrest occurred due to pulmonary thromboembolism.

\section{Discussion}

$\mathrm{GV}$ is classified by its cause and axis of rotation. According to its cause, it is divided into primary or secondary GV. Primary GV is the result of neoplasm, adhesions, or abnormalities in the attachment of the stomach. Secondary GV, however, is related to disorders of gastric anatomy or function or abnormalities (1).

According to the axis of rotation, organoaxial volvulus is most common with an incidence of $60 \%$ (2). It is most commonly associated with paraesophageal hernias and diaphragmatic eventration. GV is seldom diagnosed when a patient initially presents with upper abdominal pain or lower chest pain. After a conventional abdominal or chest radiography, GV is suspected because of the specific double air-fluid level appearance.

Chest X-ray may demonstrate a retrocardiac, air-filled mass and plain abdominal $\mathrm{X}$-ray may show a distended, fluid-filled stomach (1). Recently, abdominal CT is performed in patients who presented to the emergency department with abdominal pain due to the greater utility and easier accessibility of CT. A swirl sign, which is the appearance of rotation of the esophagus and stomach around each other on axial CT images, may be distinguished. (1). Upper gastrointestinal series with barium contrast is another useful diagnostic tool (3). Akhtar et al. (4) published a case report diagnosed with GV by using this imaging technique, although there was no sign of GV in abdominal CT.

Nasogastric tube decompression not only helps with symptoms of upper abdominal pain, but may also cause spontaneous derotation of the stomach. After resolving gastric distention, tension in the stomach wall decreases and perfusion improves. Early intensive care management, including hemodynamic monitoring and fluid resuscitation, is important because some patients present with shock or suspected intrathoracic gastric perforation due to gastric wall ischemia.

In the last decade, endoscopic detorsion has been performed in some patients with multiple comorbidities, which may worsen surgical success (5). Surgery is usually decided considering the clinical status of the patient, dimension of the defect and failure of the non-surgical interventions.

Our patient was diagnosed early and underwent surgery immediately. His clinical status improved during the postoperative follow-up period; however, on the $5^{\text {th }}$ day, unexpected sudden cardiac arrest developed. 


\section{Conclusion}

In conclusion, if a patient presents to the emergency department with abdominal pain and distention, GV should be considered in the differential diagnosis, although rare.

\section{Ethics}

Informed Consent: The patient consent form was taken from the patient's daughter.

Peer-review: Externally peer-reviewed.

\section{Authorship Contributions}

Surgical and Medical Practices: G.B., S.G., S.G., A.B.D., O.C., Concept: N.M.A., M.A., Design: N.M.A., G.B., Data Collection or Processing: G.B., S.G., I.I. ., Analysis or Interpretation: N.M.A., M.A., G.B., Literature Search: G.B., S.G., Writing: N.M.A., G.B.
Conflict of Interest: No conflict of interest was declared by the authors.

Financial Disclosure: The authors declared that this study received no financial support.

\section{References}

1. Bauman MZ, Evans CH. Volvulus. Surg Clin N Am. 2018;98:973-99.

2. McElreath DP, Olden KW, Aduli F. Hiccups: a subtle sign in the clinical diagnosis of gastric volvulus and a review of the literature. Dig Dis Sci. 2008;53:3033-6.

3. Kiyani A, Khasla M, Anufreichik V, Chuag KY. "A Large Hiatal Hernia”: Atypical Presentation of Gastric Volvulus. Clin Pract Cases Emerg Med. 2017;1:187-9.

4. Akhtar A, Siddiqui FS, Shikh AA, Sheikh AB, Perisetti A. Gastric Volvulus: A Rare Entity Case Report and Literature Review. Cureus. 2018;10:e2312.

5. Gourgiotis S, Vougas V, Germanos S, Baratsis S. Acute gastric volvulus: diagnosis and management over 10 years. Dig Surg. 2006;23:169-72. 